\title{
Religiosidade e qualidade de vida em pessoas com HIV
}

\author{
The relationship between religiousness and \\ quality of life in people living with HIV
}

\section{Bruno MEDEIROS}

Ana Alayde Werba SALDANHA'

\begin{abstract}
Resumo
Este estudo objetivou investigar a relação entre religiosidade e qualidade de vida em pessoas com vírus da imunodeficiência humana, já que a religiosidade tem sido considerada relevante nesse contexto. Participaram do presente estudo 90 pacientes soropositivos ao vírus da imunodeficiência humana de um hospital de referência em João Pessoa no estado da Paraíba, sendo 56\% do sexo masculino. Foram utilizados os instrumentos da Avaliação da World Health Organization Quality of Life Assessment Questionnaire-bref, quatro questões sobre religiosidade do World Health Organization Quality of Life Assessment Questionnaire-100 e o questionário de atitude religiosa. Foram realizadas análises descritivas e bivariadas, bem como uma análise de regressão linear múltipla (stepwise), a fim de se verificarem as variáveis que melhor explicariam a qualidade de vida nessa amostra. Ficou demonstrado que houve relação entre religiosidade e qualidade de vida entre os pacientes, ressaltando-se que a dimensão da religiosidade se relacionou com os domínios psicológico, social e ambiental de qualidade de vida, o que se manifestou de forma semelhante entre os pacientes sintomáticos. Percebe-se a importância da religiosidade no contexto da saúde e enfrentamento de doenças.
\end{abstract}

Unitermos: HIV. Religiosidade. Saúde.

\begin{abstract}
Religiousness has been cited as an important factor in the context of living with the Human Immune Deficiency Virus/Acquired Immune Deficiency Syndrome. The aim of this study was to investigate the relationship between religiousness and quality of life in people living with Human Immune Deficiency Virus/Acquired Immune Deficiency Syndrome. So 90 patients living with HIV from a reference hospital in João Pessoa, in the Brazilian state of Paraiba, participated in this study, 56\% of them being males. The World Health Organization Quality of Life Assessment Questionnaire-bref, four questions about religiousness from the World Health Organization Quality of Life Assessment Questionnaire-100 and the Religious Attitudes Questionnaire were used. Bivariate and descriptive analyses were performed as well as a multiple linear regression analysis (stepwise), that verifies which variables would best explain the quality of life in this sample. The relationship between religiousness and quality of life among the patients was demonstrated, highlighting that religiousness was related to the psychological, social and environmental domains of quality of life, which also manifested itself in similar fashion amongst symptomatic patients. The importance of religiousness in the context of health and coping with illness could be observed.
\end{abstract}

Uniterms: HIV. Religiousness. Health.

Durante as últimas décadas, o Vírus da Imunodeficiência Humana (HIV), agente causal da Síndrome da Imunodeficiência Adquirida (AIDS), tem afetado milhões de pessoas em inúmeras partes do mundo. Em 2007, de acordo com o relatório anual da World Health Organization - WHO (2007) 33,2 milhões de pessoas

vrv

1 Universidade Federal da Paraíba, Programa de Pós-Graduação em Psicologia Social, Núcleo de Pesquisa Vulnerabilidades e Promoção de Saúde. Campus I, Cidade Universitária, 58000-000, João Pessoa, PB, Brasil. Correspondência para/Correspondenceto: B. MEDEIROS. E-mail: <brunojpa@hotmail.com>. 
viviam com o vírus HIV. Estima-se que, a cada dia, mais de 6800 pessoas sejam infectadas pelo vírus HIV e mais de 5700 morram de AIDS, a maioria em virtude do inadequado acesso à prevenção, bem como da má qualidade nos serviços de tratamento. No Brasil, vivem cerca de um terço das pessoas soropositivas ao HIV na América Latina, onde a crescente política de prevenção e os serviços de tratamento têm ajudado a manter essa epidemia estável (WHO, 2008).

Com o advento dos medicamentos antirretrovirais, a AIDS passou a ser representada como uma doença crônica, assim como o diabetes e o câncer (Castanha, Coutinho, Saldanha \& Ribeiro, 2006). Nessa perspectiva, segundo Saldanha (2003), é possível delinear na história da AIDS dois momentos importantes: o período antes da década de 1990, no qual havia a imagem da AIDS relacionada à desesperança e à morte; e o período depois dessa década, com a introdução dos medicamentos antirretrovirais, proporcionando prolongamento na vida de pessoas soropositivas ao HIV e levantando-se, dessa forma, questões a respeito da qualidade de vida desse grupo de pessoas (Fleck, 2008).

Assim, estudos começaram a ser realizados a fim de avaliar os aspectos psicossociais envolvidos na vivência da soropositividade, relacionando-os à percepção de qualidade de vida, que passa a fazer parte da abordagem de políticas públicas de saúde. Esse interesse está pautado no reconhecimento dos direitos humanos e nos princípios de participação, dignidade, assistência e autorrealização das pessoas soropositivas ao HIV (Silva, Medeiros \& Saldanha, 2008; Zimpel \& Fleck, 2008).

A introdução do conceito de qualidade de vida como medida desfecho em saúde passou a existir no contexto da década de 1970, quando o grande progresso da medicina resultou em um maior prolongamento na expectativa de vida da população geral, o que refletiu em uma vivência com formas mais abrandadas ou assintomáticas das doenças. Em virtude disso, passou-se a refletir sobre a importância de se dispor de ferramentas que pudessem avaliar de que forma as pessoas vivenciariam esses anos a mais (Panzini, Rocha, Bandeira \& Fleck, 2008).

O debate sobre qualidade de vida tem crescido e abrangido desde questões referentes à relação saúde/ doença como questões socioeconômicas e ambientais.

54 Portanto, a qualidade de vida pode ser concebida como "a percepção do indivíduo de sua posição na vida no contexto da cultura e sistema de valores nos quais ele vive em relação aos seus objetivos, expectativas, padrões e especulações"(WHO, 1997). Diante disso, reconhecem-se inúmeros fatores que se correlacionam com a percepção da qualidade de vida entre as pessoas, tais como aspectos socioeconômicos, saúde física, meio ambiente, trabalho etc. (Chatterji \& Bickenbach, 2008; Rezende, 1986). Reconhece-se, dessa forma, que a noção de qualidade de vida se constitui como um fator eminentemente humano, multifacetado, sendo aproximada ao grau de satisfação encontrado na vida familiar, amorosa, social, ambiental e religiosa. Esse termo engloba diversos significados, os quais refletem conhecimentos, experiências, valores individuais e de coletividades e também diversos aspectos da vida social dos indivíduos (Minayo, Hartz \& Buss, 2000).

Reconhecendo-se que inúmeros fatores contribuem para a construção da percepção de qualidade de vida nos indivíduos, verifica-se um crescente interesse de estudo sobre o fenômeno da religiosidade como influenciadora ou não de saúde, bem como componente da qualidade de vida tanto em pessoas com estados de saúde críticos, como na população geral considerada saudável (Calvetti, Muller \& Nunes, 2008; Panzini et al., 2008).

Dessa forma, de acordo com James (1902/1995, p.31), a religiosidade pode ser definida como "os sentimentos, atos e experiências de indivíduos em sua solidão, na medida em que se sintam relacionados com o que quer que possam considerar o divino". Já Amatuzzi (2000), ao tentar compreender o fenômeno religioso, descreve a existência de um grau de enraizamento religioso, que se relaciona ao fato de que determinada forma religiosa pode ser vivenciada de diferentes maneiras, possivelmente devido às distintas crenças construídas na experiência religiosa das pessoas. Esse aspecto vem sendo estudado por diversos pesquisadores na tentativa de verificar qual a sua relação com práticas de enfrentamento de problemas e de promoção de saúde (Faria \& Siedl, 2006; Moreira-Almeida, Lotufo Neto \& Koenig, 2006).

Em virtude disso, verifica-se uma mudança de enfoque no estudo da religião, antes voltado para a simples filiação religiosa, mas agora englobando as experiências e o quanto a religião tem participado da 
vida diária das pessoas. Nesse sentido, outro importante construto relacionado ao estudo da religiosidade é o da espiritualidade, que levanta questões relativas ao significado da vida e da razão de viver, não diretamente ligadas a crenças ou práticas religiosas (Rocha, Panzini, Pargendler \& Fleck, 2008). Nessa direção, Koenig (2004) enfatiza que os construtos espiritualidade e religiosidade estão relacionados, principalmente na compreensão do fenômeno de saúde-doença. Enquanto o primeiro remete a uma dimensão de experiência mais individual, não envolvendo diretamente uma doutrina particular, moral ou princípio ético, o segundo envolve rituais, crenças e uma dimensão de fé mais comunitária.

No contexto brasileiro, a temática da religiosidade ressurge veementemente e relaciona-se com questões como aborto, adesão de tratamentos médicos e psicológicos, enfrentamento de doenças, suporte social etc. Nesse âmbito, a religiosidade torna-se fator relevante na vivência da soropositividade ao HIV, tendo em vista os resultados de pesquisas que a relacionam como possível aspecto favorecedor de bem-estar psicológico e de apoio social (Calvetti et al., 2008; Zimpel \& Fleck, 2008).

Nesse sentido, este estudo tem como objetivo investigar a relação entre a religiosidade e a percepção de qualidade de vida no contexto da soropositividade ao HIV, tendo em vista a relevância dos aspectos psicossociais presentes na maior sobrevida dessas pessoas.

\section{Método}

\section{Participantes}

Participaram desse estudo, de forma não probabilística e acidental, pessoas soropositivas ao HIV, atendidas em um hospital de referência ao atendimento de pessoas com essa infecção. $O$ período de coleta dos dados se deu no mês de abril do ano de 2010 e, como critério para participação na pesquisa, foi proposto que fossem investigados pacientes com um tempo de diagnóstico acima de um ano, tendo em vista as repercussões afetivo-emocionais do diagnóstico do HIV.

\section{Instrumentos}

Os participantes foram solicitados a responder a um conjunto de instrumentos, abaixo descritos:
Questionário sociodemográfico e clínico - suas questões tiveram por finalidade caracterizar os participantes, versando sobre: sexo, idade, grau de escolaridade, renda familiar, situação conjugal, local de residência, nível de religiosidade e dados clínicos (tempo de diagnóstico do vírus, tratamento medicamentoso, existência de doenças oportunistas etc.).

Questionário Qualidade de Vida (WHOQOLBref) - construído pela World Health Organization - WHO, o qual pressupõe que a qualidade de vida seja uma construção subjetiva (percepção do indivíduo em questão), multidimensional e composta por elementos positivos (por exemplo, mobilidade) e negativos (dor) (Fleck et al., 1999). OWHOQOL-Bref deriva da forma mais completa do Instrumento de Avaliação de Qualidade de Vida (WHOQOL-100) e tem 26 questões englobando quatro domínios: a) físico (ex. dor e desconforto e atividades da vida cotidiana), b) psicológico (ex. sentimentos positivos e autoestima), c) relações sociais (ex. relações sociais e apoio social) e d) meio ambiente (ex. recursos financeiros e cuidados de saúde e sociais).

Tendo em vista os objetivos deste estudo, foram incluídas mais quatro questões que englobam o domínio espiritualidade, religiosidade e crenças pessoais pertencente ao WHOQOL-100. Justifica-se a inclusão dessas questões em virtude de fazerem parte do instrumento da WHO e se apresentarem como importantes facetas na avaliação do construto qualidade de vida (WHO, 2006).

Questionário de Avaliação de Atitude Religiosa (QAAR) - tem por finalidade avaliar o nível de religiosidade e fé das pessoas. Elaborado por Aquino (2005), apresenta16 itens, distribuídos de acordo com os componentes da atitude afeto, cognição e comportamento - e avalia as atitudes relacionadas a eles, como: Leio as escrituras sagradas (Bíblia ou outro livro sagrado); Converso com minha família sobre assuntos religiosos; Sinto-me unido a um "Ser" maior. Esses itens são respondidos em relação ao grau de frequência das práticas e sentimentos religiosos mencionados, de acordo com uma escala que varia de nunca a sempre. Demonstra-se também uma adequação de se avaliar a religiosidade em um único fator - atitude religiosa total - com consistência interna verificada através do Alfa de Cronbach de 0,91 . 


\section{Procedimento para coleta de dados}

Após a autorização para a realização da pesquisa pelo Comitê de Ética em Pesquisa da Secretaria de Saúde do Estado da Paraíba, protocolo n 0276.0.000.349-09, em dois de março de 2009, assim como pelo hospital de referência no atendimento a pessoas soropositivas ao HIV, foi iniciada a fase de coleta de dados, em que os participantes foram informados, previamente, a respeito dos objetivos e procedimentos da pesquisa, bem como da confiabilidade dos dados e do anonimato da sua colaboração. Foi solicitado aos participantes que lessem e assinassem um termo de consentimento informado, cujo modelo foi elaborado de acordo com a Resolução n¹96/96 Sobre Pesquisa Envolvendo Seres Humanos (Brasil, 1996). Caso os participantes não soubessem ler, o investigador teria a responsabilidade de ler e explicar o termo de consentimento informado, de forma a deixar claras as suas intenções ao realizar a pesquisa. Dessa forma, os pacientes foram entrevistados nas enfermarias e também no Serviço de Atendimento Especializado (SAE), onde era promovida a assistência especializada.

\section{Análise dos dados}

Os dados foram submetidos a análises descritivas e bivariadas (correlação). Os escores do questionário WHOQOL-Brefforam transformados em índices com ponderação de 0 a 100, em que 0 corresponde ao menor e 100 ao maior valor encontrado para cada domínio, tendo em vista os parâmetros psicométricos desta escala. Foi utilizado o Teste $t$ de Student para verificar as diferenças entre as médias dos grupos-critérios. Para que fosse demonstrado o significado da força e do tipo de relação existente entre as dimensões estudadas, foi utilizado o coeficiente de correlação r de Pearson, acompanhado do seu respectivo nível de significância ( $p)$. Além disso, foi realizada uma análise de regressão linear múltipla (stepwise) com o objetivo de verificar quais variáveis melhor explicariam a qualidade de vida para a presente amostra. Nesse sentido, a partir da revisão da literatura sobre a vivência com o HIV, foram incluídos os aspectos psicossociais e físicos como variáveis independentes para uma melhor avaliação de qualidade de vida (variável dependente); dentre esses aspectos, este estudo privilegiou os domínios que contribuem para a avaliação de qualidade de vida pela World Health
Organization (físico, psicológico, social, ambiental), a religiosidade/espiritualidade e os determinantes clínicos da infecção pelo HIV (contagens de células CD4).

\section{Resultados}

Participaram deste estudo 90 pacientes de um hospital de referência no atendimento de pessoas soropositivas ao HIV, na faixa etária de 19 a 48 anos, sendo $56 \%$ do sexo masculino, com média de idade de 34 anos (DP=6,6); destes, 64\% possuíam nível de escolaridade até o ensino fundamental. Dos pacientes entrevistados, 63\% residiam na área metropolitana de João Pessoa (João Pessoa, Santa Rita, Cabedelo e Bayeux) e 52\% estavam empregados. Com relação à renda familiar, $81 \%$ recebiam até dois salários-mínimos. Em relação à religião, 60\% dos pesquisados se autodenominaram católicos; 18\%, evangélicos; 11\%, mencionaram pertenceram à outra religião/forma de espiritualidade e 11\% se autodenominaram sem religião. Observou-se também que a respeito da avaliação de quanto os pacientes se consideravam religiosos, a maioria se denominou "Religiosa" (33\%) e"Muito Religiosa" (30\%).

Em relação aos dados clínicos, o tempo de diagnóstico dos pacientes variou de 1 até 28 anos $(M=6,25, D P=4,91)$. Verificou-se também que $88 \%$ faziam uso da terapia antiretroviral e 60\% relataram não terem contraído doenças oportunistas. A contagem dos linfócitos T, CD4, (números de células por milímetro cúbico de sangue), indicadora das condições do sistema imunológico, variou desde 32 até $1250 \mathrm{~mm}^{3}$, apresentando uma média de 557,42 por $\mathrm{mm}^{3}(\mathrm{DP}=248,19)$.

Já em relação aos dados psicossociais, verificou-se que a percepção da qualidade de vida pelos pesquisados foi positiva $(M=65, D P=19,9)$, ainda que próxima ao ponto de corte para uma avaliação negativa $(M=50)$, percebendo-se avaliações satisfatórias nos domínios físico $(M=59,56, D P=17,81)$, psicológico $(M=70,64$, $D P=15,61)$, social $(M=65,83, D P=22,72)$, religiosidade/ espiritualidade e crenças pessoais ( $M=76,53, D P=16,21)$ e ambiental $(M=56,66, D P=15,75)$ de qualidade de vida, constatando-se que as mulheres se avaliaram melhor fisicamente do que os homens ( $t=2,29, \mathrm{gl}=82,9, p=0,02)$. Além desses resultados, foi realizada uma análise de regressão linear múltipla (stepwise) com o objetivo de 
verificar quais variáveis melhor explicariam a qualidade de vida para a presente amostra (Tabela 1).

A partir desses resultados, constatou-se que a dimensão psicológica, a maior contagem de células CD4 e o domínio ambiental foram as variáveis que melhor explicaram a avaliação satisfatória quanto à qualidade de vida para os participantes deste estudo. Percebeu-se ainda que a associação entre essas variáveis e avaliação de qualidade de vida (variável dependente) foi moderadamente forte ( $R$ múltiplo=0,71). Juntas, o domínio psicológico, a maior contagem de células CD4 e o domínio ambiental, explicaram 46\% da variância total da avaliação subjetiva de qualidade de vida.

A média de atitude religiosa total demonstrada pelo QAAR foi de 64,71 (DP=15,17), observando-se uma correlação positiva entre o domínio religiosidade/espiritualidade e crenças pessoais e a avaliação de qualidade de vida geral $(r=0,33 ; p=0,001)$. Investigando-se quais domínios da qualidade de vida se correlacionaram com religiosidade, percebeu-se que o domínio religiosidade/espiritualidade e crenças pessoais se correlacionou com os domínios: psicológico $(r=0,39 ; p=0,001)$, social $(r=0,41 ; p=0,001)$ e ambiental $(r=0,35 ; p=0,001)$ do WHOQOL-Bref. Contudo, não foi percebida relação entre atitude religiosa total e os domínios de qualidade de vida; entretanto, a atitude religiosa total se correlacionou positivamente com o domínio religiosidade/espiritualidade e crenças pessoais ( $r=0,30 ; p=0,003)$, percebendo-se também correlação positiva entre atitude religiosa total e a avaliação sobre "o quanto você se considera religioso?" $(r=0,56 ; p=0,001)$

No grupo de pacientes sintomáticos $(n=35$ foi verificada uma correlação positiva entre o domínio religiosidade/espiritualidade e crenças pessoais e qualidade de vida geral $(r=0,47 ; p=0,004)$, percebendo-se também que o domínio religiosidade/espiritualidade e crenças pessoais esteve relacionado com os domínios social $(r=0,58 ; p=0,001)$, psicológico $(r=0,58 ; p=0,001)$ e ambiental $(r=0,35 ; p=0,03)$ de qualidade de vida. Ressalta-se ainda uma correlação entre domínio religiosidade/ espiritualidade e crenças pessoais e atitude religiosa total $(r=0,37 ; p=0,02)$ entre os pacientes sintomáticos.

\section{Discussão}

Nos dias atuais, percebe-se uma maior incidência de casos de HIV em populações mais pobres economicamente e em um contexto de fatores estruturais de risco, bem como de escassez de recursos. Nessa direção, no presente estudo, foi verificada essa tendência social e econômica da contaminação pelo HIV, pois a amostra reflete um contexto socioeconômico de pobreza. Corroborando esses resultados, Parker e Camargo Jr. (2000) enfatizam que o subdesenvolvimento econômico e a pobreza constituem-se fatores intimamente relacionados à proliferação do vírus HIV, pois a pandemia global da AIDS tem se desenvolvido concomitante às mudanças sociais e econômicas as quais tem construído espaços de exclusão social e econômica e promovido maior vulnerabilidade para que a infecção ocorra. Ressalta-se, portanto, que pessoas soropositivas HIV, além da condição de enfermidade, lidam com carências sociais e econômicas que seguramente têm impacto na percepção de qualidade de vida (Siedl, Zannon \&Trócolli, 2005).

Neste estudo, a predominância masculina de pacientes entrevistados (1,25 homens para cada mulher) reflete a tendência brasileira, pois os homens ainda apresentam-se como a maioria de infectados pelo HIV, entretanto essa diferença vem diminuindo ao longo dos anos de epidemia no Brasil, verificando-se, por exemplo, que a razão de sexo (masculino: feminino) passou de 15,1:1 em 1986 para 1,5:1 em 2006 (Brasil, 2008). Aspecto esse que, de acordo com Guimarães (2001), reflete o processo de feminização da doença.

Tabela 1. Análise de regressão múltipla com as variáveis com maior poder explicativo para o escore de qualidade de vida geral.

\begin{tabular}{lcccccc}
\hline Variáveis & $R$ & $R^{2}$ & $F$ & Sig(F) & Beta & T \\
\hline Psicológico & 0,515 & 0,241 & $11,16(31)$ & 0,002 & 0,394 & 2,94 \\
CD4 & 0,639 & 0,369 & $10,34(30)$ & 0,0001 & 0,420 & 3,18 \\
Ambiental & 0,715 & 0,460 & $10,09(29)$ & 0,0001 & 0,328 & 2,46 \\
\hline
\end{tabular}

${ }^{*}$ R2 apresentado é acumulado e ajustado. 
A maior expectativa de vida percebida pode refletir a eficácia do tratamento com os antirretrovirais, o que se constitui aspecto primordial tanto para o tratamento de pessoas soropositivas ao HIV como para o desenvolvimento de pesquisas a respeito dessa problemática, o que favorece não apenas o aumento da sobrevida dos pacientes, mas também preocupações sobre questões psicossociais envolvidas com a vivência com o HIV (Saldanha, 2003).

A terapia antirretroviral foi introduzida no sistema de saúde brasileiro a partir de 1996, como parte da política de acesso universal e gratuito aos serviços de saúde e aos medicamentos; entretanto a sua eficácia tem se relacionado com a sua adesão (Melchior, Nemes, Alencar \& Buchalla, 2007), assim como com as avaliações subjetivas - positivas ou negativas - dos pacientes a respeito da eficácia de seu tratamento (Remor, 2002). Nesse contexto, a correta administração do tratamento tem contribuído não apenas para a melhoria da saúde física, mas também em outras dimensões da vida dos pacientes, como na avaliação de qualidade de vida das pessoas soropositivas ao HIV (Fleck, 2008). Confirmando esse aspecto, Santos, França Junior e Lopes (2007), em um estudo realizado em um serviço ambulatorial de atendimento a pessoas vivendo com HIV, no qual foi utilizado o WHOQOL-Bref, perceberam que, dentre os principais fatores sociodemográficos e clínicos relacionados com a avaliação de qualidade de vida, destacaram-se: sexo, presença de sintomas, tempo conhecido de infecção pelo HIV e contagens de células CD4.

Nessa direção, demonstrou-se uma boa avaliação de qualidade de vida entre os pacientes deste estudo, assim como em seus diversos domínios, corroborando resultados de estudos que expõem a relevância da dimensão da qualidade de vida, principalmente em um contexto de doença crônica (Aranha-Naranjo, 2004; Den Oudsen, van Heck, van der Steeg, Roukema \& De Vries, 2008).

As questões de gênero perpassam a dinâmica de cuidado com a saúde, o que se evidencia pela melhor avaliação das mulheres em relação a sua dimensão física, o que se constitui importante no caso da soropositividade ao HIV, já que as mulheres vêm sofrendo com o aumento de casos infectados bem como com as consequências da doença para seu convívio familiar e social 58 (Amaro, 2005). Além disso, a verificação de que as questões psicológicas, a contagem de células CD4 e os aspectos ambientais (recursos financeiros, estruturais e de cuidados à saúde) melhor explicaram a percepção de qualidade de vida entre os participantes vem ratificar um estudo realizado na China, em que os aspectos psicossociais tiveram peso na explicação de qualidade de vida entre as pessoas vivendo com HIV, já que as experiências de estigmatização e preconceito pareciam influenciar negativamente a dinâmica familiar na obtenção de trabalho estável, assim como na procura por atividades de lazer, aspectos esses que se relacionavam com a dimensão psicológica e a ambiental de qualidade de vida (Meng et al., 2008).

Na mesma direção, em um estudo, realizado no Brasil, com o objetivo de validar o questionário de Qualidade de Vida para pessoas com HIV (WHOQOL-HIV), verificou-se que, com o avanço da doença, o agravamento clínico da infecção repercute no corpo e na capacidade de autogerenciamento das pessoas, o que levanta a relevância da relação entre sistema imunológico e aspectos psicossociais (Zimpel \& Fleck, 2008).

A noção de qualidade de vida avança no sentido de avaliar diversas dimensões envolvidas com a saúde (Fleck et al., 1999), ressaltando-se que a religiosidade presente entre as pessoas soropositivas demonstra a sua interface na avaliação de qualidade de vida (Castanha, Coutinho, Saldanha \& Ribeiro, 2007), sendo observado que a dimensão religiosidade/espiritualidade se encontrava relacionada à avaliação de qualidade de vida geral entre os pacientes. Esse resultado corrobora pesquisas que demonstram que as dimensões religiosa, espiritual e de crenças pessoais se encontram relacionadas mais com a qualidade de vida em pessoas com situações de saúde comprometidas (Fleck, Borges, Bolognesi \& Rocha, 2003; WHO, 2006).

Reconhece-se, portanto, que a dimensão religiosidade perpassa os dilemas da condição de doença (Tournier, 1984) e é importante fator no enfrentamento de doenças como a AIDS (Panzini \& Bandeira, 2005). De forma semelhante, Siedl (2005), ao investigar estratégias de enfrentamentos de pessoas vivendo com HIV, verificou que a busca por práticas religiosas é uma das estratégias mais utilizadas.

A relação entre religiosidade e os domínios psicológico, social e ambiental de qualidade de vida pode se referir ao papel da religiosidade como favorecedora de bem-estar psicológico e apoio social e estrutural a 
pessoas acometidas por doenças e outras condições clínicas (Ai, Tice, Huang, Rodgers e Bolling, 2008; Fleck et al., 2003; Moreira-Almeida et al., 2006). Nesse contexto, destaca-se a relevância do apoio social característico de muitos sistemas religiosos, que possibilita auxílio em momentos de sofrimento (Lotufo Neto, 2003). Por exemplo, no contexto da soropositividade ao HIV, em que, muitas vezes, a pouca participação da família e a pouca rede de apoio social contribuem para a "morte social" da pessoa, destaca-se o papel da religiosidade e dos centros religiosos como aspectos confortadores (Silva et al., 2008). De forma semelhante, no grupo de pacientes sintomáticos, foi também verificada relação entre religiosidade/espiritualidade e os domínios psicológico, social e ambiental de qualidade de vida, o que demonstra a presença da religiosidade no manejo da dor e dos cuidados paliativos (Peres, Arantes, Lessa \& Caous, 2007), ressaltando-se que a religiosidade tem-se demonstrado presente no manejo e enfrentamento de doenças crônicas (Oliveira, Fernandes \& Galvão, 2005; Vachon, 2008).

Entretanto, a falta de relação entre a atitude religiosa e os domínios de qualidade de vida pode significar que, para essa amostra, embora exista a presença marcante da religiosidade, as dimensões mais significativas para a percepção de qualidade de vida foram a psicológica, a contagem de células CD4 e as questões ambientais e estruturais, como foi demonstrado pela análise de regressão. Contudo, percebeu-se que, quanto mais os participantes consideravam-se religiosos, mais demonstraram afeto, cognição e comportamentos religiosos, o que se relaciona com a literatura em questão, que verifica que o comprometimento religioso tem repercutido em ações de enfrentamento de problemas e de promoção de saúde (Faria \& Siedl, 2006; Moreira-Almeida et al., 2006).

Em virtude disso, reconhece-se que a religiosidade pode ser considerada uma dimensão doadora de sentido à experiência humana cotidiana. Há certo consenso entre estudiosos de diversas áreas de que a religião é uma importante instância de significação e ordenação da vida, de seus reveses e sofrimentos. Nesse sentido, como elemento constitutivo da subjetividade e doador de significado ao sofrimento, torna-se relevante estudá-la no campo de saúde e percepção de qualidade de vida, principalmente em um contexto de doença (Dalgalarrondo, 2008).

\section{Considerações Finais}

A problemática da infecção pelo HIV apresenta, para o campo da saúde, desafios e oportunidades. A percepção da relevância dos aspectos psicossociais na sobrevida de pessoas soropositivas ao HIV favorece a elaboração de políticas públicas que alcancem esses aspectos. Nesse sentido, reconhece-se que o estudo da religiosidade e da espiritualidade como possibilidade de enfrentamento em um contexto de doença crônica pode auxiliar profissionais de saúde no tratamento e planejamento de ações voltadas para pessoas vivendo com HIV, tendo em vista o reconhecimento de que condições clínicas, psicológicas, sociais e espirituais perpassam os dilemas vivenciados por essas pessoas.

Todavia, reconhecem-se as limitações do presente estudo em se aprofundar nas variáveis psicossociais relacionadas ao maior prolongamento de vida de pessoas vivendo com HIV, tendo em vista a dimensão da amostra pesquisada e o foco do estudo. Portanto, recomendam-se novas pesquisas para avaliar melhor a influência da religiosidade no enfrentamento da condição de doença e sugere-se a realização de estudos que englobem as questões de gênero envolvidas com o cuidado com a saúde, pois, a partir deste estudo, constataram-se diferenças entre homens e mulheres na avaliação física relacionada à qualidade de vida.

\section{Referências}

Ai, A. L., Tice, T. N., Huang, B., Rodgers, W., \& Bolling, S. F. (2008). Types of prayer, optimism, and well-being of middle-aged and older patients undergoing open-heart surgery. Mental Health, Religion and Culture, 11 (1), 131-150.

Amaro, S. T. A. (2005). A questão da mulher e a Aids: novos olhares e novas tecnologias de prevenção. Saúde e Sociedade, 14 (2), 89-99.

Amatuzzi, M. M. (2000). Desenvolvimento religioso: uma hipótese psicológica. Estudos de Psicologia (Campinas), $17(1), 15-30$.

Aquino, T. A. A. (2005). Atitude religiosa e crenças dos alunos de psicologia. Revista do UNIPÊ, 9 (1), 56-63.

Aranha-Naranjo, B. (2004). Quality of e Life in the HIVpositive patient: implications and consequences. Journal of the Association of Nurses in Aids Care, 15 (5), 20-27.

Brasil. Ministério da Saúde. (1996). Resolução 196/96 sobre pesquisa envolvendo seres humanos. Brasília: CNS.

Brasil. Ministério da Saúde (2008). Secretaria de Vigilância em Saúde. Programa Nacional de DST e AIDS. In Boletim epidemiológico de AIDS/DST: Série A, n.84. Brasília: Autor. 
Calvetti, P. U., Muller, M. C., \& Nunes, M. L. T. (2008). Qualidade de vida e bem-estar espiritual em pessoas vivendo com HIV/AIDS. Psicologia em Estudo, 13 (3), 523-530.

Castanha, A. R., Coutinho, M. P. L., Saldanha, A. A., \& Ribeiro, C. G. (2006). Repercussões psicossociais da depressão no contexto da Aids. Psicologia Ciência e Profissão, 26 (1), 70-81.

Castanha, A. R., Coutinho, M. P. L., Saldanha, A. A., \& Ribeiro, C. G. (2007). Avaliação de qualidade de vida em soropositivos para o HIV. Estudos de Psicologia (Campinas), 24 (1), 23-31. doi: 10.1590/S0103-166×2007000100003.

Chatterji, S., \& Bickenbach, J. (2008). Considerações sobre a qualidade de vida. In M. P. A. Fleck (Org.), A avaliação de qualidade de vida: guia para profissionais da saúde (pp.40-47). Porto Alegre: Artmed.

Dalgalarrondo, P. (2008). Religião, psicopatologia e saúde mental. Porto Alegre: Artmed.

Den Oudsen, B. L., van Heck, G. L., van der Steeg, A. F. W., Roukema, J. A., \& De Vries, J. (2008). The WHOQOL-100 has good psychometric properties in breast cancer patients. Journal of Clinical Epidemiology, 62 (2), 195-205.

Faria, J. B., \& Siedl, E. M. F. (2006). Religiosidade, enfrentamento e bem-estar subjetivo em pessoas vivendo com HIV/AIDS. Psicologia em Estudo, 11 (1), 155-64.

Fleck, M. P. A. (2008). Problemas conceituais em qualidade de vida. In M. P. Fleck (Org), A avaliação de qualidade de vida: guia para profissionais da saúde (pp.19-28). Porto Alegre: Artmed.

Fleck, M. P. A., Borges, Z. N., Bolognesi, G., \& Rocha, N. S. (2003). Desenvolvimento do WHOQOL, módulo espiritualidade, religiosidade e crenças pessoais. Revista de Saúde Pública, 37 (4), 446-55.

Fleck, M. P. A., Leal, O. F., Louzada, S., Xavier, M., Chachamovich, E., \& Vieira, G. (1999). Desenvolvimento da versão em português do instrumento de avaliação de qualidade de vida da OMS (WHOQOL-100). Revista Brasileira de Psiquiatria, 21 (1) 19-28.

Guimarães, C. D. (2001). Aids no feminino: por que a cada dia mais mulheres contraem Aids no Brasil? Rio de Janeiro: UFRJ.

James, W. (1995). As variedades da experiência religiosa: um estudo sobre a natureza humana. São Paulo: Cultrix. (Originalmente publicado em 1902).

Koenig, H. G. (2004). Spirituality, wellness, and quality of life. American Society for Reproductive Medicine, 2 (2), 76-82.

Lotufo Neto, F. (2003). Religião, psicoterapia e saúde mental. In C. N. Abreu (Org.), Psicoterapias cognitiva e construtivista: novas fronteiras da prática clínica. Porto Alegre: Artmed.

Melchior, R., Nemes, M. I. B., Alencar, T. M. D., \& Buchalla, C. M. (2007). Desafios da adesão ao tratamento de pessoas vivendo com HIV/Aids no Brasil. Revista de Saúde Pública, 41 (2), 87-93.

Meng, Y. J., Li, N. X., Liu, C. J., Chen, J. H, Song, Y. C., \& Qian, Z. S. (2008). Quality of life and hostile mentality trend of patients with HIV/AIDS in China. The Royal Institute of Public Health, 122 (4), 404-411

Minayo, M. C. S., Hartz, Z. M. A., \& Buss, P. M. (2000). Qualidade de vida e saúde: um debate necessário. Ciência \& Saúde Coletiva, 5 (1), 7-18.

Moreira-Almeida, A., Lotufo Neto, F., \& Koenig, H. G. (2006). Religiousness and mental health: a review. Revista Brasileira de Psiquiatria, 28 (3), 242-250.

Oliveira, M. S., Fernandes A. F. C., \& Galvão, M. T. G. (2005). Mulheres vivenciando o adoecer em face do câncer cérvico-uterino. Acta Paulista de Enfermagem, 18 (2), 150-5.

Panzini, R. G., \& Bandeira, D. R. (2005). Escala de coping religioso-espiritual (escala CRE): elaboração e validação de construto. Psicologia em Estudo, 10 (3), 507-516.

Panzini, R. G., Rocha, N. S., Bandeira, D. R., \& Fleck, M. P. A. (2008). Espiritualidade/religiosidade e qualidade de vida. In M. P. A. Fleck (Org.), A avaliação de qualidade de vida: guia para profissionais de saúde (pp.177-96). Porto Alegre: Artmed.

Parker, R., \& Camargo Jr., K. R (2000). Pobreza e HIV/AIDS: aspectos antropológicos e sociológicos. Caderno de Saúde Pública, 16 (1), 89-102.

Peres, M. F. P., Arantes, A. C. L. Q., Lessa, P. S., \& Caous, C. A. (2007). A importância da integração da espiritualidade e da religiosidade no manejo da dor e dos cuidados paliativos. Revista Psiquiatria Clínica, 34 (1), 82-87.

Remor, E. A. (2002). Aspectos psicossociais na era dos novos tratamentos da AIDS. Psicologia: Teoria e Pesquisa, 18 (2), 283-287.

Rezende, A. L. M. (1986). Saúde, dialética do pensar e do fazer. São Paulo: Cortez.

Rocha, N. S., Panzini, R. G., Pargendler, J. S., \& Fleck, M. P. A. (2008). Desenvolvimento do módulo para avaliar espiritualidade, religiosidade e crenças pessoais do WHOQOL (WHOQOL-SRPB). In M. P. A. Fleck. A avaliação de qualidade de vida: guia para profissionais de saúde (pp.93-101). Porto Alegre: Artmed.

Saldanha, A. A. W. (2003). Vulnerabilidade e construções de enfrentamento da soropositividade ao HIV por mulheres infectadas em relacionamento estável. Tese de Doutorado não-publicada, Programa de Pós-Graduação em Psicologia, Faculdade de Filosofia, Ciências e Letras de Ribeirão Preto, Universidade de São Paulo.

Santos, E. C. M., França Junior, I., \& Lopes, F. (2007). Qualidade de vida de pessoas vivendo com HIV/Aids em São Paulo. Revista de Saúde Pública, 41 (2), 64-71.

Seidl, E. M. F. (2005) Enfrentamento, aspectos clínicos e sociodemográficos de pessoas vivendo com HIV/AIDS. Psicologia em Estudo, 10 (3), 421-429.

Seidl, E. M. F., Zannon, C. M. L. C., \& Trócolli, B. T. (2005). Pessoas vivendo com HIV/ AIDS: enfrentamento, suporte social e qualidade de vida. Psicologia Reflexão e Crítica, 18 (2), 188-195. 
Silva, J., Medeiros, B., \& Saldanha, A. A. W. (2008). "Morte e morrer" e qualidade de vida: a influência da participação social e da religiosidade na sobrevida de pessoas acima de 50 anos HIV+. Anais do IX Congresso Virtual HIV/AIDS, Ciência Social e Comportamental. Lisboa, Portugal.

Tournier, P. (1984). Culpa e graça: uma análise do sentimento de culpa e o ensino do evangelho. São Paulo: ABU.

Vachon, M. L. S. (2008). Meaning, spirituality, and wellness in cancer survivors. Seminars in Oncology Nursing, 24 (3), 218-225

World Health Organization. (1997). WHOQOL measuring quality of life. Programme on Mental Health. Division of Mental Health and Prevention of Substance Abuse. Washington, DC: Author.

World Health Organization. (2006). A cross-cultural study of spirituality, religion, and personal beliefs as components of quality of life. Social Science \& Medicine, 62 (6), 1486-1497.

World Health Organization. (2007, December). AIDS epidemic update. Washington, DC: Author.

World Health Organization. (2008). Latin America: AIDS epidemic update: regional summary. Washington, DC: Author.

Zimpel, R., \& Fleck, M. P. A. (2008). Qualidade de vida em pacientes com HIV/AIDS: conceitos gerais e resultados de um estudo brasileiro. In M. P. A. Fleck (Org.), A avaliação de qualidade de vida: guia para profissionais da saúde (pp.157-67). Porto Alegre: Artmed.

Recebido em: 13/1/2010

Versão final reapresentada em: 14/6/2011

Aprovado em: 29/6/2011 
